\title{
REDESIGN DA JORNADA DE COMPRA EM UM CANAL DIGITAL UTILIZANDO PRINCÍPIOS DO DESIGN DE EXPERIÊNCIA
}

Felipe Teixeira Braz (felipetbraz.07@gmail.com) - Pontifícia Universidade Católica de Minas Gerais (PUC-Minas).

Marianna Barros Badaró (marianna.badaro@hotmail.com) - Pontifícia Universidade Católica de Minas Gerais (PUC-Minas).

Matheus Luiz Pontelo de Souza (mslzposa@gmail.com) - Pontifícia Universidade Católica de Minas Gerais (PUC-Minas).

\section{RESUMO}

Devido à crescente busca por parte das empresas e instituições pela melhoria de seus sites e/ ou produtos digitais para entregar uma melhor experiência de uso aos seus clientes e usuários, fidelizando-os, várias empresas vêm investindo recursos em criar um produto que seja atrativo e que entregue ao usuário a maior facilidade em termos de navegação e manuseio. Princípios de design de experiência apresentam potencial de contribuição para que estes resultados sejam alcançados. Este trabalho tem como objetivo apresentar os resultados obtidos no número de conversões após a aplicação de princípios e métodos de design de experiência. As ferramentas como Google Analytics, CRM Hubspot e Whatsapp foram utilizadas para a coleta de dados deste caso e, como resultados, foi percebida melhora significativa no indicador de qualidade da jornada de compras do cliente. Considerando que o comportamento do usuário se altera conforme sua jornada de atendimento, este estudo evidencia - a partir de um caso - como uma melhor usabilidade acarreta melhores resultados.

Palavras-chave: design de experiência, usabilidade, experiência de usuário, produtos digitais, melhoria 


\section{INTRODUÇÃO}

O desenvolvimento de produtos digitais tem mostrado uma demanda crescente na área de Engenharia de produto, as empresas vêm investindo no desenvolvimento de sites, aplicativos e sistemas próprios, a fim de sanar problemas e de oferecer novas funcionalidades para os seus clientes. A utilização de um site, aplicativo ou sistema pode ajudar as empresas na padronização do atendimento, na melhoraria da experiência do cliente oferecendo assim outros benefícios.

É necessário entender o que de fato motiva o usuário a utilizar o produto e o que ele está buscando para entregar um produto que possa proporcionar ao usuário uma experiência única e altamente relevante (CASTRO, 2019). Para o caso específico de um site é preciso facilitar a interação com o produto, já que as pessoas tendem a interagir mais com aquilo que é prático para elas. Conhecer o cliente tende a melhorar a capacidade da empresa de eleger recursos inteligentes e alinhados com as demandas das pessoas que forem utilizar o programa. Desde a linguagem visual até o tipo de mecanismo de segurança, tudo será montado a partir das necessidades do seu público-alvo.

Buscando entender como melhorar a experiência do cliente durante o processo de compra, focado em uma maior conversão, foram pesquisados métodos, ferramentas e princípios com potencial de aplicação para melhorar o design de experiência na jornada de compra de um produto digital. Após a avaliação, estes foram aplicados na melhoria do design de experiência de um site já existente, buscando uma navegabilidade facilitada e intuitiva, e que ao mesmo tempo consiga fazer com que o engajamento do usuário seja maximizado.

Os autores utilizaram como caso de aplicação o site utilizado por uma instituição de ensino voltada à venda de cursos. Este site é utilizado como ferramenta de geração de leads e conversão de clientes. $\mathrm{O}$ foco principal do estudo foi a melhoria no processo de atendimento ao cliente, que é iniciado no site e concluído através de outros canais. A aplicação deste estudo visou a melhoria do design de experiência do site, a fim de aumentar o número de conversões realizadas durante a jornada de compras em análise.

\section{REVISÃO TEÓRICA}

Segundo Brown (2019), a jornada do cliente é estruturada a partir de um mapeamento dos estágios pelos quais um cliente passa do início ao fim de sua experiência com um serviço ou 
produto. A estruturação citada por Brown (2019) é feita através da identificação dos "pontos de contato" com o pretendido cliente, nos quais existem oportunidades de fornecer valor ao mesmo em cada um desses pontos, sendo estes pontos, fatores definidores para atrair ou não os clientes. Outro fator de relevância na jornada do cliente, principalmente quando se trata de um serviço digital, é a usabilidade.

A ISO/IEC 25000 (2014) define a usabilidade da seguinte forma: "Grau em que um produto ou sistema pode ser usado por usuários específicos para atingir metas especificadas com eficácia, eficiência e satisfação em um contexto específico de uso.". Segundo a ISO 25000 (2014), a usabilidade é composta por seis sub características, sendo elas: reconhecimento de adequabilidade, aprendizagem, operacionalidade, proteção contra erros do usuário, estética da interface do usuário e acessibilidade.

Preece, Rogers e Sharp (2013) apontam alguns atributos como os principais definidores da usabilidade de um produto, são eles: (1) Eficiência: o produto deve apresentar um alto nível de produtividade depois de o usuário se familiarizar com o mesmo. (2) Eficácia: o produto deve ser bom em fazer aquilo que se espera dele. (3) Segurança: o sistema deve ser modelado de forma com que os possíveis erros do usuário sejam minimizados, e caso eles venham a ocorrer, que causem o menor dano possível e sejam facilmente recuperáveis. (4) Utilidade: o sistema deve fornecer funcionalidades que satisfaçam os desejos e necessidades dos usuários. (5) Satisfação: o prazer sentido pelo usuário (subjetivamente) durante e após o uso do produto.

Sob o ponto de vista do design de produtos digitais, a importância de cada um dos atributos acima varia de acordo com o tipo de produto que está sendo desenvolvido, sua finalidade, perfil dos usuários, entre outros fatores. A aplicação dos atributos apontados por Preece, Rogers e Sharp (2013) pode ser um fator decisivo no desempenho do produto e na sobrevivência da empresa como um todo. Uma empresa de ecommerce por exemplo, que tem o seu site como a ferramenta de vendas e, consequentemente, de faturamento, precisa que o seu site tenha um bom nível de usabilidade para que os usuários se deparem com o menor número possível de problemas na navegação. Isto porque ao se depararem com problemas de usabilidade, a primeira reação do usuário, na maioria das vezes, será a de deixar o site, num cenário em que existem vários outros sites que fornecem aquele mesmo produto. Doherty e Sorenson (2015) apontam seis fatores como os mais influentes na performance percebida por 
um usuário em uma interface digital. Os fatores apontados por Doherty e Sorenson (2015) são: (1) Background, que pode ser resumidamente entendido como o tempo que o sistema leva para responder a uma ação do usuário, (2) Flow, o fluxo de interação do usuário com o sistema deve conter o menor número de interrupções, (3) Percepção do tempo, onde eles argumentam que o tempo de espera para processos como downloads e uploads passa a ser diferente do tempo esperado pelo usuário quando ele ultrapassa os limites de sensibilidade do usuário, (4) Atenção, em que são citadas quais ações prendem mais a atenção de cada tipo de usuário, (5) Feedback, o sistema deve reduzir a incerteza dos usuários e passar para o mesmo a sensação de que ele está no controle e que o sistema está agindo de acordo com as ações do usuário, (6) Influência da percepção, onde são citados as expectativas do usuário, complexidade percebida e os limites da percepção humana como os três princípios psicológicos para a percepção do usuário.

Por meio de elementos artísticos que despertam emoções no usuário, o profissional de design de experiência consegue atrair o usuário através dessa emoção gerada, antes mesmo do produto mostrar sua utilidade para o usuário. Além disso, a emoção pode influenciar nas tomadas de decisão do usuário durante o uso do produto (NORMAN, 2008). O profissional de design de experiência pode guiar essas emoções através de, cores e formatos de componentes da interface, elementos neuro linguísticos, sons e etc.

Finalmente, ciclos de teste e mensuração de resultados - mesmo que simples - são um elemento fundamental do design de experiência (HARTSON; PYLA, 2012). Os autores afirmam ainda que usabilidade ou experiência não são conceitos diretamente mensuráveis, sendo necessário usar indicadores indiretos sobre a percepção de satisfação do usuário com sua experiência. Exemplos citados são indicadores de tempo e produtividade (HARTSON; PYLA, 2012) que, no contexto deste trabalho, podem ser indicadores de conversão em uma jornada do usuário ou de conversão em testes de duas opções (teste A/B).

\section{METODOLOGIA}

Este trabalho classifica-se como uma pesquisa-ação (COUGHLAN; COGHLAN, 2002) cujo objetivo foi melhorar a experiência do usuário por meio do monitoramento de seu comportamento durante uma jornada que se inicia na navegação do site e é concluída em outros canais. 
Tal abordagem envolveu desafios vivenciados pela empresa da qual um dos pesquisadores faz parte, e seguiu os quatro passos de Eden e Ackermann (2018) para elaboração de pesquisaação envolvendo aplicação de métodos/princípios - como, neste caso, os de usabilidade/design de experiência. Os passos foram: a compreensão dos princípios da perspectiva da aplicação; o design da aplicação prática em seguida à aplicação no contexto da prática para extrair valor da aplicação em si e de suas implicações e, finalmente, o quarto passo, envolvendo os ciclos típicos da pesquisa-ação - neste caso evidenciados por experimentos (EDEN; ACKERMANN, 2018). A Figura 2 oferece maior detalhamento destes passos.

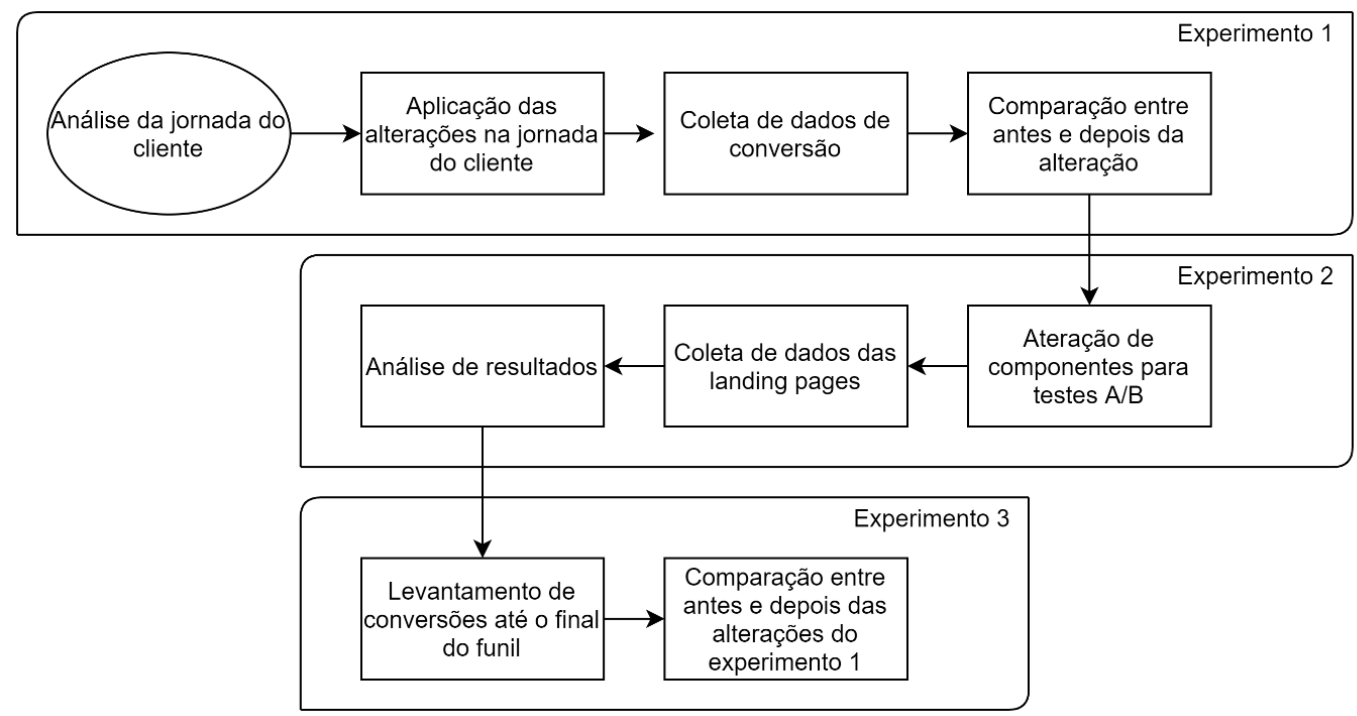

FIGURA 2 - Etapas da metodologia. Fonte: Autores

Os ciclos envolveram a aplicação de princípios e métodos de design de experiências e coleta de dados que envolveram posicionamento de componentes nas páginas, tamanho e tipo de fontes utilizadas, cores e contrastes de componentes e fontes, tipos de informações dispostas no site, forma com que as informações estavam dispostas no site, caminho que o usuário deveria percorrer para atingir o seu objetivo, dentre outros atributos.

Para a realização do experimento 1 , o número de conversões das páginas foi coletado do CRM (Customer Relationship Management, que pode ser traduzido para Gestão de Relacionamento com o Cliente) Hubspot, em que o site envia automaticamente para o CRM as informações de todos os usuários que preenchem formulários. No primeiro momento, no painel de atividades da equipe, foi utilizado apenas o filtro geral de atividades dos últimos 365 dias que se refere ao filtro máximo disponível. No segundo momento, na aba de contatos, 
foram utilizados os filtros "data de criação" entre 15 de fevereiro de 2021 e 23 de março de 2021, e "fonte original" como nenhuma offiline. Após a coleta das informações no CRM, foi realizado manualmente, um levantamento de conversão de clientes e do tempo de atendimento para cada cliente no histórico de conversas do Whatsapp da instituição, para assim ser calculado o tempo para atendimento.

A realização do experimento 2, iniciou-se utilizando os testes $\mathrm{A} / \mathrm{B}$, que consistem em encaminhar metade dos usuários de uma campanha de marketing para uma página, e a outra metade dos usuários para uma página quase idêntica. Essas duas páginas têm como diferença apenas um componente, podendo ser a forma como uma informação é passada, a ausência ou presença de uma informação, o posicionamento de um botão, a cor de um botão, e qualquer outro componente que possa influenciar as ações do usuário.

Com a ferramenta do teste A/B, foi possível comparar diferentes disposições de uma página, sendo possível assim, classificar a alteração de componente como positiva ou negativa. $\mathrm{O}$ teste $\mathrm{A} / \mathrm{B}$ aplicado no trabalho teve uma diferença para os testes $\mathrm{A} / \mathrm{B}$ tradicionais, enquanto os testes A/B tradicionais fazem os experimentos de duas versões ao mesmo tempo, neste trabalho, cada teste foi aplicado em um período diferente.

Em seguida, foi utilizada a ferramenta Google Analytics, que além de várias outras funcionalidades, fornece o número de visualizações e usuários que uma página recebeu durante determinado período. Assim, com os dados dos períodos estudados e com os contatos convertidos adquiridos no experimento anterior, foram calculadas as taxas de conversão. Já o experimento 3, a coleta de usuários foi feita da mesma maneira que no experimento 2, entretanto a taxa de conversão foi calculada até o final do funil. Todo o tratamento de dados retirados das ferramentas citadas anteriormente foi feito pelo programa Microsoft Excel.

\section{RESULTADOS E DISCUSSÃO}

Na escola técnica em que o trabalho de melhoria de design de experiência foi aplicado, a jornada do cliente até receber o atendimento ocorria da seguinte forma, ao entrar no site o cliente poderia receber o atendimento de três maneiras: (1) entrar em contato através de ligação telefônica, pelo contato exposto no topo do site, (2) clicar no link de redirecionamento para o WhatsApp, (3) preenchendo um formulário de contato. Antes de aplicar os testes, os autores realizaram um desenho do fluxo da jornada do cliente, detalhado na figura 3. 


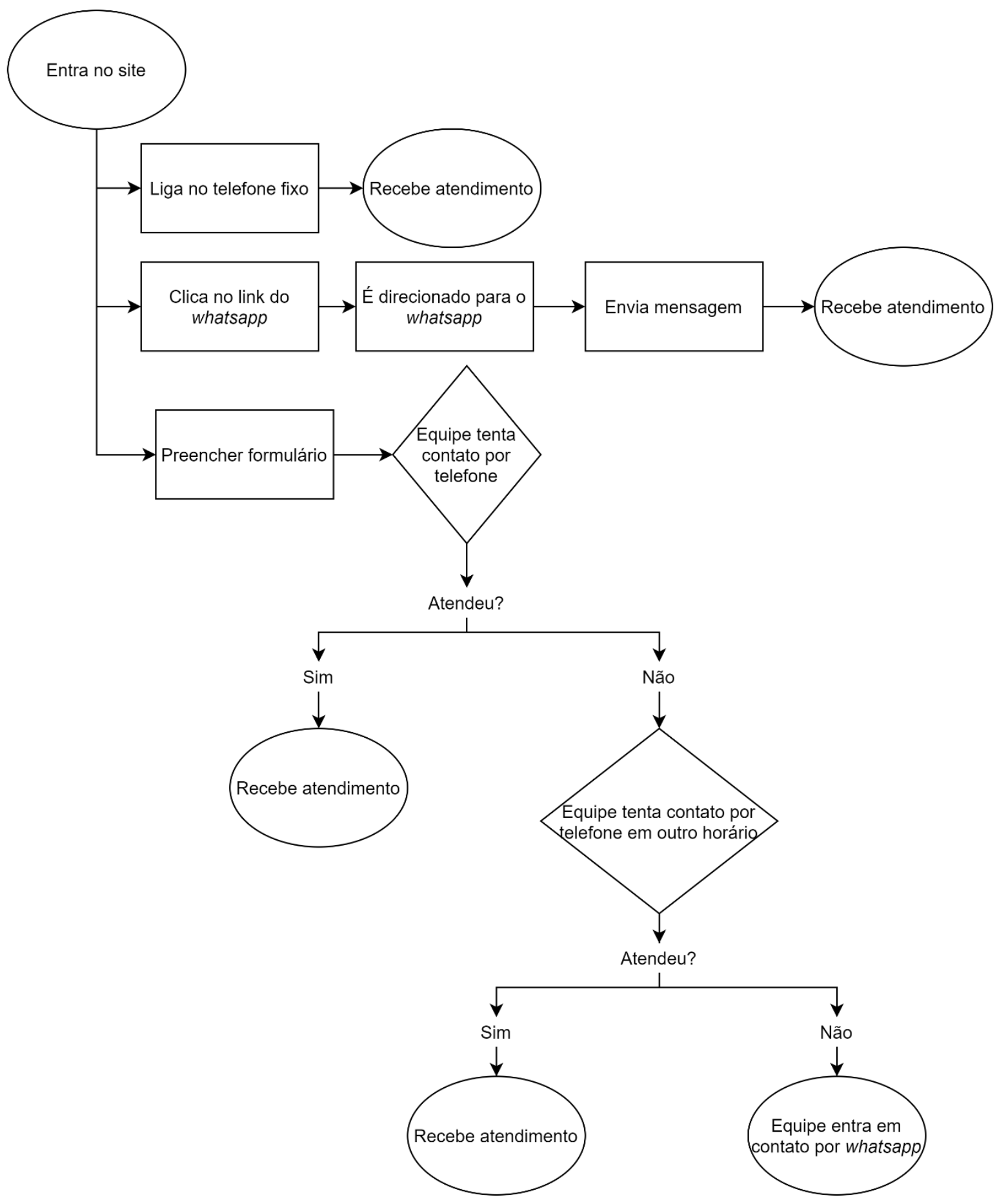

FIGURA 3 - Jornada do cliente até receber o atendimento. Fonte: Autores

\subsection{Alteração de jornada de atendimento}

No início do ano de 2021, devido à sazonalidade do segmento, ocorreu um pico de procura por cursos técnicos, e a equipe, que já trabalhava de forma enxuta, estava levando muito 
tempo para realizar os atendimentos de clientes que preenchiam formulários. Além do tempo gasto para registrar as chamadas no software de CRM, muitas das ligações não eram atendidas. Em um levantamento feito pela equipe de pesquisa entre as datas de 24 de março de 2020 e 22 de março de 2021, os resultados obtidos são apresentados na Tabela 1.

TABELA 1 - Levantamento de chamadas realizadas.

Tipos de chamadas Número de chamadas Porcentagem

\begin{tabular}{ccc}
\hline Chamadas realizadas & 998 & $100 \%$ \\
\hline $\begin{array}{c}\text { Chamadas com atendimento realizado } \\
\begin{array}{c}\text { Chamadas em que o cliente pediu } \\
\text { para retornar em outro horário }\end{array}\end{array}$ & 567 & $57 \%$ \\
\hline Chamadas não atendidas & 45 & $5 \%$ \\
\hline $\begin{array}{c}\text { Chamadas sem atendimento realizado } \\
\text { (Soma das linhas 3 e 4) }\end{array}$ & 431 & $39 \%$ \\
\hline
\end{tabular}

A alta taxa de chamadas sem atendimento realizado estava fazendo com que a equipe de atendimento gastasse muito tempo para realizar o atendimento. Além disto, a equipe também levava certo tempo para registrar tais chamadas no CRM. Visando agilizar o atendimento dos contatos que preenchiam formulários, a equipe passou a atender estes clientes diretamente pelo Whatsapp antes de tentar contato por ligação, conforme detalhado na Figura 4.

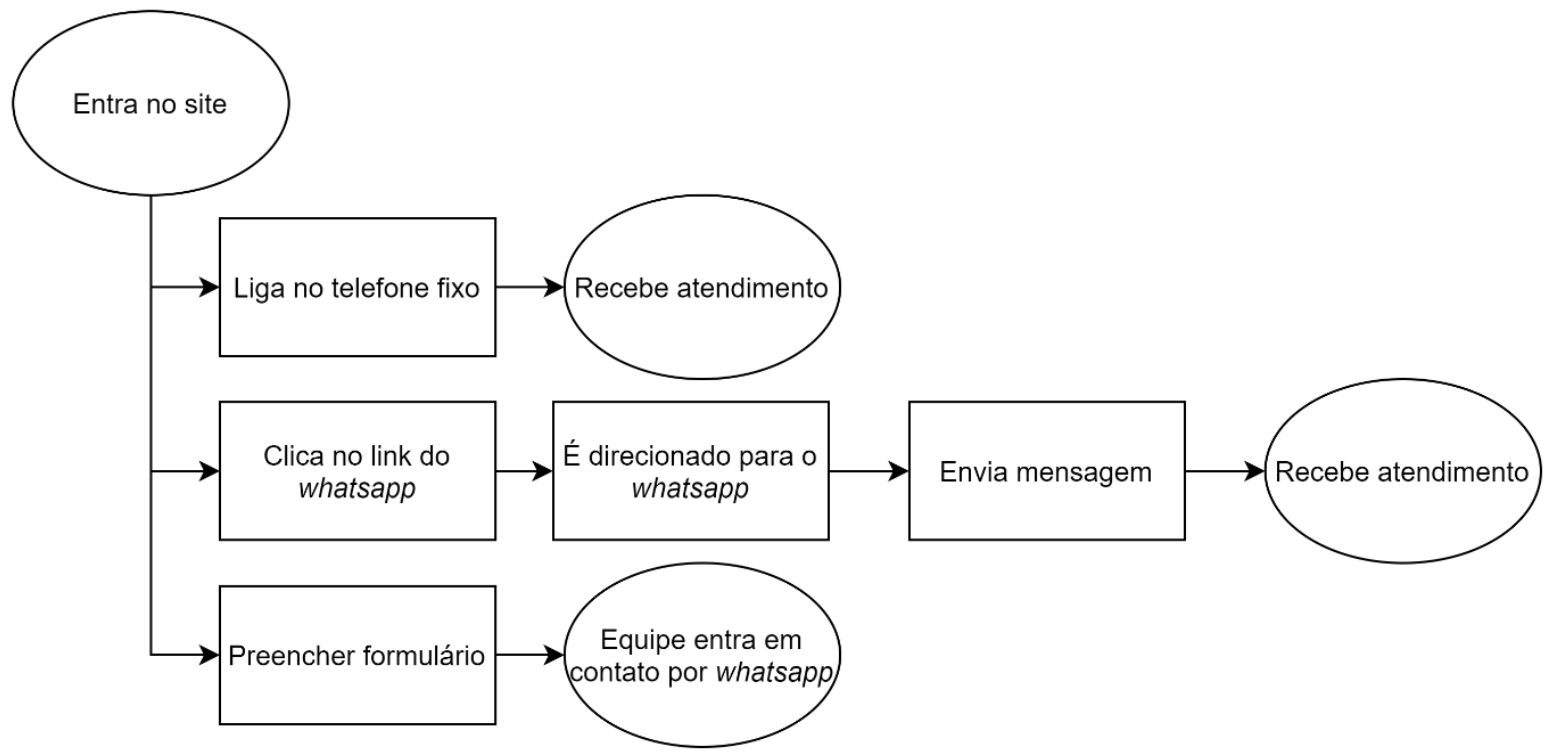

FIGURA 4 - Jornada do cliente após alteração. Fonte: Autores 
Ainda com esta mudança, a equipe estava levando um tempo longo para realizar o atendimento aos clientes que preenchiam formulários, com isso, muitos clientes já estavam com o interesse menor quando recebiam o atendimento. A partir daí, os autores da pesquisa fizeram um levantamento de dados para relacionar o tempo de atendimento com a porcentagem de clientes que dão retorno ao atendimento. Foram coletados dados entre as datas de 15 de fevereiro de 2021 e 23 de março de 2021, e conforme detalhado na Tabela 2, foi constatado que quanto maior o tempo para o atendimento (tempo entre o envio do formulário e o primeiro contato da equipe de atendimento com o cliente), menor era a taxa de conversão de clientes para as próximas fases do funil de vendas.

TABELA 2 - Relação entre tempo de atendimento e conversão.

\begin{tabular}{cccc} 
Tempo até atendimento & $\begin{array}{c}\mathbf{N}^{\circ} \text { de clientes } \\
\text { atendidos }\end{array}$ & $\begin{array}{c}\mathbf{N}^{\circ} \text { de clientes que } \\
\text { deram retorno }\end{array}$ & Aproveitamento \\
\hline Até 1 hora & 21 & 19 & $90 \%$ \\
\hline Entre 1 e 4 horas & 6 & 5 & $83 \%$ \\
\hline Entre 4 e 24 horas & 18 & 16 & $89 \%$ \\
\hline Entre 24 e 72 horas & 20 & 10 & $50 \%$ \\
\hline Mais de 72 horas & 48 & 29 & $60 \%$ \\
\hline
\end{tabular}

Foi observado um comportamento em alguns dos clientes que poderia indicar a solução para este problema. Estes clientes estavam preenchendo o formulário de contato e, logo depois, entrando em contato pelo Whatsapp, desta forma, tendo uma conversão maior para as próximas fases do funil de venda. A Tabela 3 mostra a diferença de conversão entre os clientes que preencheram o formulário e em seguida entraram em contato por Whatsapp e aqueles que preencheram o formulário e aguardara o retorno da instituição.

TABELA 3 - Comparação de diferentes jornadas de cliente.

\begin{tabular}{cccc} 
Ações tomadas pelo cliente & $\mathbf{N}^{\mathbf{0}}$ de clientes & $\begin{array}{c}\mathbf{N}^{\circ} \text { de clientes que } \\
\text { deram retorno }\end{array}$ & Aproveitamento \\
\hline Preencheu formulário & 76 & 46 & $61 \%$ \\
\hline $\begin{array}{c}\text { Preencheu formulário e entrou em } \\
\text { contato por whatsapp }\end{array}$ & 37 & 33 & $89 \%$ \\
\hline
\end{tabular}


A partir disto, foram aplicadas algumas alterações na jornada dos clientes que preenchiam formulários, foram elas: (1) aplicação de um ícone de Whatsapp no cabeçalho do formulário de contato, (2) Alteração do texto do formulário de contato de "Vamos conversar" para “Consulte valores, datas e horários", que são as informações mais buscadas pelos clientes, e (3) Alteração do destino dos clientes que preenchiam formulários, que eram direcionados para a página de obrigado, e passaram a ser direcionados para o Whatsapp.

Após as alterações nos formulários de contato, a equipe realizou um levantamento dos clientes que entraram em contato entre as datas de 1 de abril de 2021 e 24 de abril de 2021, já com o novo formato de formulário integrado ao site. Foi constatado que a média de tempo para atendimento caiu de 72 horas para 46 horas, uma melhoria de aproximadamente 36\%, com isso, o percentual de clientes que passaram para as etapas seguintes do funil subiu de $70 \%$ para $73 \%$. Apesar da melhora no aproveitamento, pode se julgar que o tempo para atendimento não é o único fator que afeta a conversão de clientes, visto que, em proporção, a melhora no aproveitamento foi significativamente menor que a melhora no tempo para atendimento.

Além do indicador citado acima, outro indicador apresentou uma melhora significativa. A conversão de usuários que visitam o site por preenchimento de formulários ficou quase duas vezes maior, passando de $6 \%$ para $11 \%$. O número de clientes que entraram em contato diretamente por Whatsapp ou telefone, se comparado ao número de acessos do site nos respectivos períodos também apresentou uma melhora, passando de $14 \%$ para $22 \%$. No entanto, não é possível afirmar que essa melhora está diretamente ligada às alterações no site, já que os clientes que entram em contato diretamente pelo Whatsapp ou telefone podem ser oriundos de outros canais de divulgação.

\subsection{Teste de componentes}

Para entender a influência de cada alteração do formulário na melhora das conversões de visitantes, a equipe testou duas delas de forma separada, em diferentes landing pages (também conhecidas como páginas de captura, são páginas que contém informações de um produto/serviço, onde a única ação que o cliente pode tomar é a de preencher o formulário). Na primeira landing page, foi testado um formulário apenas com o símbolo do Whatsapp no cabeçalho. Já na segunda, o cabeçalho do formulário continha somente a frase "Consulte valores, datas e horários". Outras três landing pages similares foram testadas com os dois 
componentes alterados. O experimento foi realizado entre as datas de 19 de abril de 2021 e 7 de maio de 2021. A Tabela 4 mostra a evolução no número de conversão das páginas.

TABELA 4 - Conversões das landing pages.

\begin{tabular}{|c|c|c|c|}
\hline Alteração & $\begin{array}{c}\text { Conversão antes } \\
\text { da alteração }\end{array}$ & $\begin{array}{c}\text { Conversão depois } \\
\text { da alteração }\end{array}$ & $\begin{array}{c}\text { Aumento da } \\
\text { conversão }\end{array}$ \\
\hline $\begin{array}{l}\text { Simbolo do Whatsapp no } \\
\text { cabeçalho }\end{array}$ & $27 \%$ & $41 \%$ & $52 \%$ \\
\hline Frase com informações & $43 \%$ & $58 \%$ & $35 \%$ \\
\hline As duas alterações juntas & $13 \%$ & $33 \%$ & $154 \%$ \\
\hline
\end{tabular}

Observando a melhora nas conversões gerada por cada alteração, pode se presumir que o interesse pelas informações principais (valores, datas e horários) faz com que os clientes passem para as fases seguintes do funil. Também foi possível presumir que os clientes foram mais atraídos quando o formulário continha o símbolo de Whatsapp.

\subsection{Efetividade das alterações no final do funil de vendas}

Ficou evidente nos experimentos 1 e 2, que as alterações realizadas foram positivas para as conversões do site. No entanto, ainda não havia sido verificado se essas alterações estavam fazendo diferença nos resultados do processo de venda. Por isso, foi feito um levantamento do percentual de clientes que se matricularam para os cursos técnicos.

Foram comparados os clientes que entraram em contato (por todos os canais) nos mesmos períodos do Experimento 1, entre 15 de fevereiro e 23 de março e entre 1 e 24 de abril, que foram detalhados respectivamente nas Tabelas 5 e 6 . Os clientes que procuraram cursos que não estavam com matrículas abertas não foram incluídos no cálculo.

TABELA 5 - Conversão de clientes de 15 de fevereiro a 23 de março de 2021.

\begin{tabular}{lcccc} 
Estágio & $\mathbf{N}^{0}$ de usuários & Quantidade Conversão & Conversão acumulada \\
\hline Estágio 1 & Usuários no site & 1522 & - & - \\
\hline Estágio 2 & Usuários convertidos & 257 & $16,90 \%$ & $16,90 \%$ \\
\hline Estágio 3 & Negócios fechados & 35 & $13,60 \%$ & $2,30 \%$ \\
\hline
\end{tabular}

TABELA 6 - Conversão de clientes de 01 a 24 de abril de 2021. 


\section{Estágio $\mathrm{N}^{\circ}$ de usuários Quantidade Conversão Conversão acumulada}

\begin{tabular}{lcccc}
\hline Estágio 1 & Usuários no site & 463 & - & - \\
\hline Estágio 2 & Usuários convertidos & 135 & $29,20 \%$ & $29,20 \%$ \\
\hline Estágio 3 & Negócios fechados & 16 & $11,90 \%$ & $3,50 \%$ \\
\hline
\end{tabular}

Comparando os números de conversões dos dois períodos, pode se observar que, apesar da taxa conversão do estágio 2 para o estágio 3 ter diminuído após as alterações, a conversão acumulada ainda se manteve maior em aproximadamente 50\%. Como a conversão do estágio 1 para o estágio 2 aumentou em aproximadamente $73 \%$ depois da alteração, já era esperado que a conversão do estágio 2 para o estágio 3 fosse menor, presumindo que parte dos usuários que passaram a ser convertidos depois da alteração, foram usuários com um nível de interesse menor.

\section{CONCLUSÃO}

No início deste estudo, os autores traçaram os modelos de jornada do cliente antes das aplicações de alterações. A partir disto, foi observado o comportamento de usuários que preenchiam formulários e entravam em contato por Whatsapp imediatamente depois e, a partir disto, sugeriu-se um possível caminho para melhorias, reduzindo o tempo para atendimento em 36\%. Devido a uma inesperada melhora no número de conversões de usuários no primeiro experimento, os autores decidiram realizar o segundo experimento redesenhando a jornada do cliente a partir de princípios de usabilidade, voltados para a jornada e o fluxo de vendas, a fim de atestar qual das alterações na interface gráfica era responsável pelo aumento das conversões.

O terceiro experimento serviu para atestar a efetividade das alterações nos resultados do setor comercial da instituição de ensino. A equipe comercial da instituição relatou que a melhoria foi além dos resultados imediatos gerados. O aumento da taxa de conversão de contatos do site, mesmo que não acompanhado proporcionalmente do aumento da taxa de conversão de negócios fechados, é importante para a realização de divulgações orgânicas pelo Whatsapp e, dessa forma, estes clientes podem vir a fechar negócio futuramente.

Os métodos aplicados neste trabalho podem inspirar aplicações em outras realidades. Para a aplicação dos métodos, foi necessário o uso de ferramentas de armazenamento de dados e 
histórico de ações dos usuários no site que, no caso, foram o programa Google Analytics e o CRM Hubspot, mas podem ser utilizadas quaisquer ferramentas que realizem as mesmas funções destas. Além da utilização das ferramentas, também é preciso se certificar de que a equipe comercial da empresa faça os registros manuais de informações de forma correta para que se tenha uma mensuração precisa das ações dos usuários.

O presente artigo conseguiu identificar várias mudanças de comportamento dos usuários referente as aplicações das alterações no site e na jornada de atendimento como um todo. No futuro, estudos semelhantes podem ser focados em diferentes métodos de design de experiência como entrevistas com os usuários que, devido ao contexto de pandemia, não puderam ser aplicados neste trabalho. As entrevistas podem ter como foco o entendimento dos motivos que levaram os usuários às mudanças de comportamentos que, no presente estudo, não puderam ser identificados ou puderam ser apenas presumidos com os métodos aplicados. Desta forma, serão capturadas outras percepções e melhorias necessárias e, consequentemente, o aumento da compra.

\section{REFERÊNCIAS}

CASTRO, B. Reações afetivas na interação de usuários com componentes de produtos digitais. [S.l.]: Universidade Federal de Campina Grande. 2019.

BROWN, Tim. Change by Design - How Design thinking transforms organizations and inspires innovation. HarperCollins ebooks. 2009.

COUGHLAN, Paul; COGHLAN, David. Action research for operations management. International journal of operations \& production management, 2002.

EDEN, Colin; ACKERMANN, Fran. Theory into practice, practice to theory: Action research in method development. European Journal of Operational Research, v. 271, n. 3, p. 1145-1155, 2018.

FLEURY, A.; NAKANO, D.; CORDEIRO, José H. D. (coord.). Mapeamento da indústria brasileira de jogos digitais. São Paulo: BNDES, 2014. Disponível em: <https://www.bndes.gov.br/wps/wcm/connect/site/807f877e22d7-44e5-b11c-

c6bd1cf65b66/chamada_publica_FEP0211_mapeamento_da_industria.pdf?MOD=AJPERES\&CVID=1xKa6Gq> . Acesso em: 22 set. 2020.

HARTSON, Rex; PYLA, Pardha S. The UX Book: Process and guidelines for ensuring a quality user experience. Elsevier, 2012.

ISO - INTERNATIONAL ORGANIZATION FOR STANDARDIZATION. ISO 9241-11: Ergonomic requirements for office work with visual display terminals: Guidance on Usability. 1998.

ISO/IEC 25000:2014. Software engineering - System and software Quality Re-quirements and Evaluation (SQuaRE). Genebra: ISO, 2014.

JONGWOOK, J.; NEUnGHOE, K.; PETER, I. Detecting Usability Problems in Mobile Applications on the Basis of Dissimilarity in User Behavior. International Journal of Human-Computer Studies, v. 139, 2019. 
LIN, C. H. Research in the Use of Product Semantics to Communicate Product Design Information. [S. $l$.]: Human Centered Design, p.556-565, 2011.

MINGE, Riedel. meCUE - Ein modularer Fragebogen zur Erfassung des Nutzungserlebens, p.89-98, 2013.

NORMAN, D. Design emocional: por que adoramos (ou detestamos) os objetos do dia-a-dia. Tradução de Ana Deiró. Rio de Janeiro: Rocco, 2008.

PREECE, J.; ROGERS, Y.; SHARP, H. Design de interação: além da interação homem-computador. Porto Alegre: Bookman, 2013.

RUSSELL, J.A., WEISS, A., MENDELSOHN, G.A. Affect grid: a single-item scale of pleasure and arousal. J. Pers. Soc. Psychol. 57 (3), p. 493-502, 1989.

TRUKENBROD, A.; BACKHAUS, N.; THOMASCHKE, R. Measuring Subjectively Experienced Time in Usability and User Experience Testing Scenarios. International Journal of Human-Computer Studies, v. 138, 2020 .

HARTSON, Rex; PYLA, Pardha S. The UX Book: Process and guidelines for ensuring a quality user experience. Elsevier, 2012.

NIELSEN, Jakob. A 100-Year View of User Experience. Nielsen Norman Group. Disponível em: < https://www.nngroup.com/articles/100-years-ux/>. Acesso em: 22 mai. 2021

DOHERTY, Rina A.; SORENSON, Paul. Keeping users in the flow: Mapping system responsiveness with user experience. Procedia Manufacturing, v. 3, p. 4384-4391, 2015.

INVISION. 2019 Product Design Hiring Report. Nova Iorque. 2019. Disponível em: <https://www.invisionapp.com/hiring-report>. Acesso em: 21 mai. 2021. 